\title{
Ontology and Rule-Based Recommender System for E-learning Applications
}

https://doi.org/10.3991/ijet.v14i15.10566

\author{
Bouchra Bouihi ( $\left.\varpi^{\prime}\right)$, Mohamed Bahaj \\ University Hassan 1st, Settat, Morocco \\ bouchrabouihi@gmail.com
}

\begin{abstract}
The continuous growth of the internet has given rise to an overwhelming mass of learning materials. Which has increased the demand for a recommendation system to filter information and to deliver the learning materials that fit learners learning context. In this paper, we propose an architecture of a semantic web based recommender system. The proposed architecture is a redesigned architecture of the classical 3-tiers web application architecture with an additional semantic layer. This layer holds two semantic subsystems: an Ontology based subsystem and SWRL (Semantic Web Rule Language) rules one. The Ontology subsystem is used as a reusable and sharable domain knowledge to model the learning content and context. The SWRL rules are used as a recommendation and filtering technique based on learning object relevance and weighting. These rules are organized into four categories: Learning History Rules (LHR), Learning Performance Rules (LPR), Learning Social Network Rules (LSNR) and Learning Pathway Rules (PR).
\end{abstract}

Keywords-Semantic web, Recommendation System, Ontology, E-learning, SWRL.

\section{$1 \quad$ Introduction}

E-learning is a new form of distance learning that relies on providing educational content via internet or intranet. These contents are organized into modules that can be assembled into training courses. E-learning offers a great flexibility of use for the learner who can train at his own pace according to his needs and his availability.

The combination of different multimedia contents (texts, dynamic illustrations, voice-over, videos ...) creates rich, attractive and effective work environments, having a strong impact on learner's comprehension and memorization. Interactivity can also stimulate the interest of the learner, especially during self-correcting exercises.

In spite of that, the endless development of e-learning systems has engendered an enormous quantity of learning materials spread over the world wide web. Consequently, it is tedious for learners to come across the suitable learning materials that fit their needs. Indeed, Recommendation systems are the key to filter data and recommend relevant learning materials. 
Better handling the learning context is the key role to offer a fruitful learning experience to learner by recommending the suitable learning materials. Contextual information like; prerequisite, interests, social interactions, and activity history; are a guide to recommend to learners the required learning materials ( $\mathrm{Yu}$ et al.). The personalisation of e-learning platforms gives to learners the chance to have a learning experience adapted to their needs. This new learning paradigm is known as context-aware elearning applications (Schmidt and Winterhalter).

The relevance of context-aware e-learning applications depends on the performance of the adopted context modelling technique. Hence, ontology could be the best choice to model the context. It has the capacity to represent a complex context knowledge domain provided with formal semantics. The ontology-based representation gives the opportunity to share and integrate context information (Villalon et al.).

Most of current widespread e-learning platforms are developed according the 3-tier architecture. This later is composed of three layers : the presentation layer, the application layer linked to the server, and he data layer linked to the database server. One of the 3-tier architecture advantages is more flexibility in ressources allocation; the portability of the third-party server makes it possible to envisage an allocation or a dynamic modification according to evolving needs, also a great flexibility for the introduction of any new technologies like semantic web technology. For this purpose we propose in this work a revised version of this architecture to incorporate a semantic layer that hosts an ontology and rule based approach for semantic recommendation systems.

The main objective of our approach is to use the ontology as a domain knowledge for modeling both learning content and context information and to explore the SWRL Rules filtering as a recommendation technique. The filetring stage is based on the learning object relevance that is determined by the learning object's weight. This later will be updated after every SWRL rule application. These rules are organised into four categories : Learning History Rules (LHR), Learning Performance Rules (LPR), Learning Social Network Rules (LSNR) and Learning Pathway Rules (PR).

The paper is organised as follows. Section 2 outlines the state of the art on semantic recommendation systems. Section 3 explains the proposed architecture. And, section 4 summerizes the paper.

\section{Related Works}

Exploring the semantic web technologies especially ontology in personalized learning has interested many researchers (Benlamri and Zhang, 2014; Shishehchi et al.). Different ontologies-based approaches for context-aware and recommendation elearning platforms were proposed. The work presented by ( $\mathrm{Yu}$ et al.) proposes to make learning content recommendations by realizing learner context-awareness in its different features; user context, content, and the learning domain. To achieve this goal, they conceived three ontologies by focusing in the recommendation process on learner's prior knowledge and his learning objective. However, they didn't take into 
consideration the learner social interactions that gives additional important contextual information.

(Shen and Shen) proposed a recommendation learning content system based on experts learning object knowledge base and personal learning progress. Sequencing rules are used to make the connection between learning objects. They are calculated from the knowledge base and the competency gap. However, the attention is only given to learning content without taking into consideration the learner profile and its social interactions that are crucial context information.

(Henze et al.) Presented a framework to realize personalization in an ontologybased e-learning system. The framework hosts: user ontology, observation ontology, and domain ontology. Reasoning mechanism are used over distributed Resource Description Framework (RDF) annotation. In this work, they used Triple as a query rule language. However, OWL is powerfully expressive than RDF.

(Shishehchi et al.) Presented a semantic recommendation system for e-learning applications. It's composed of two subsystems; ontology based subsystem and (OWL) rules subsystem. In this work, it's not explained how the ontology is built and which Rule language is used.

The added value of our work in comparison with the previous mentioned works is that, we propose a revised architecture of the 3-tier architecture that is widely used in most current e-learning platforms. We propose to add a semantic layer that will host the semantic recommendation system that is consisted of two subsystems: LMS Ontology Subsystem and SWRL Rules Subsystem.

\section{Related Works}

Most of current widespread e-learning platforms are developed according the 3-tier architecture. This later is composed of three layers : the presentation layer, the application layer linked to the server, and the data layer linked to the database server. These solutions are not sufficiently aware of the learner context. The contextawareness is highly recommended to deliver to the learner the learning material relevant to the current situation of the learner. To achieve this, we adopt the ontological approach to define a model to represent and manage context information. In this work, we want to perform a re-engineering of this architecture, with a view to incorporate the technology of the Semantic Web. To this end, we are proposing to insert a Semantic Layer between the layer of data and that of the application. The communication between these layers is ensured by the mapping between them. Figure 1 resumed schematically this architecture.

The added layer is organized in two main parts :

- LMS Ontology

- Semantic Rule 


\subsection{The LMS ontology}

Our approach is mainly based on semantic modeling of learner's learning context. To achieve this objective, we make use of semantic web technologies with a special focus on ontologies. The LMS ontology is designed to be the Learning Management System ontology. Taking into account that we work on current e- learning platforms which in great part are conceived with UML diagrams and with the purpose of minimizing the efforts needed to build such a colossal ontology, we propose to build this ontology by opting for the UML-To-OWL approach proposed in (Bouihi and Bahaj). Hence, the resulting OWL ontology will be modified so that the learner's context can be correctly modeled. To make the modeling task more manageable, we subdivide this ontology into two sub-ontologies: an ontology to model the learning content and an ontology to model the learning context.

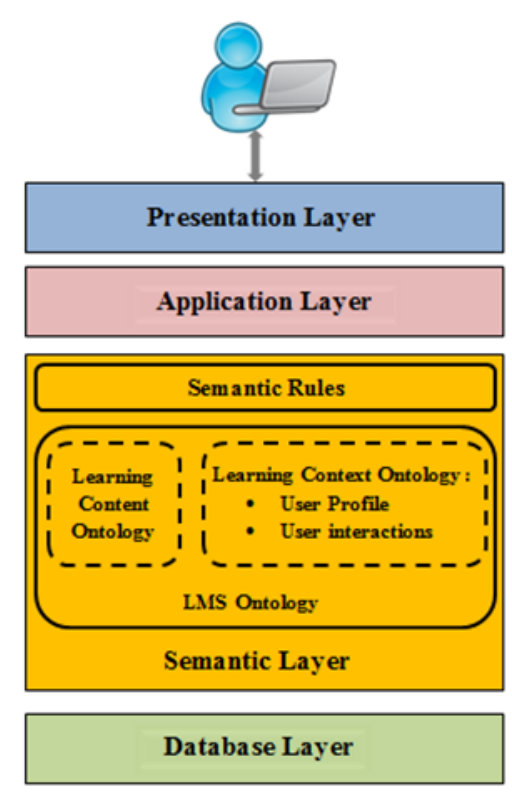

Fig. 1. A Semantic Web-based Architecture for an e-learning recommendation system

The Learning Content Ontology: Learning Objects are small size digital constituent part of a learning course that can be reusable in various learning contexts. Therefore, a learning content can be defined as a Learning Object instance. Although, these Learning Objects are usually conceived by different institutions and authors which makes the learning content semantically incoherent. This incoherence influences its reusability. Indeed, it is crucial to think about a common modeling of Learning Objects to make them accessible, reusable shareable and semantically interoperable.

To improve learning systems and modeling related learning objects, different standards have been developed. Employing these standards not only ensures interoperability, but also the quality of the system (Grandbastien et al.). These standards 
include LOM, SCORM and IMS-LD. LOM is interested in the description of the learning content, SCORM in the content structure, and the IMS-LD in the learning process scenario. In this paper, we focus our interest in the LOM standard. LOM (Learning Object Metadata) is a standard promoted by the IEEE consortium. It's used to define the structure of a metadata instance for a Learning Object. It's consisted of a set of 80 elements organized into 9 categories each fulfilling a different function (Soualah-Alila et al.). To assimilate Learning Objects semantics, we represent this standard ontologically in figure 2.

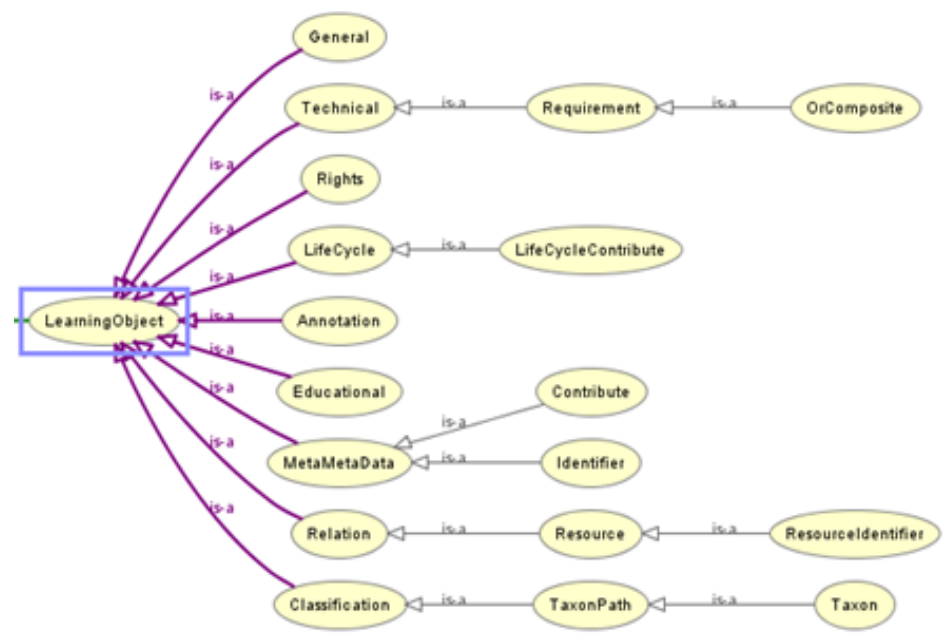

Fig. 2. The LOM standard Ontology

The Learning Context Ontology: Learning context is a fundamental feature of elearning. Indeed, it is crucial to determine, depending on the current context of the learner, what are the relevant and suitable learning materials to recommend, how and when. The entire learning process must be context-specific. To consider the context in an e-learning system, it is mandatory to represent it. This representation must have a consistent model for storing and processing context information to respond to context changes. Semantically, we represent context information by making use of a learning context ontology that comprises two interdependent sub-ontologies: learner profile and social interaction ontology and learner activity ontology. This ontology will gather all learning context information.

Figure 3 presents learner profile and social interactions Ontology that is inspired from FOAF ontology. FOAF Ontology is an ontology that is designed basically on the Resource Description Framework RDF2. It's designed to represent people's personal information and social interactions and relationships within a social network. People are represented as nodes and relationships as edges (Buriano).

FOAF is a widespread ontology. (Ding et al.) states that the class foaf: Person has approximately one million instances spread across 45,000 web documents. It is therefore useful to reuse it to represent contextual information about the learner's profile and social interactions. 


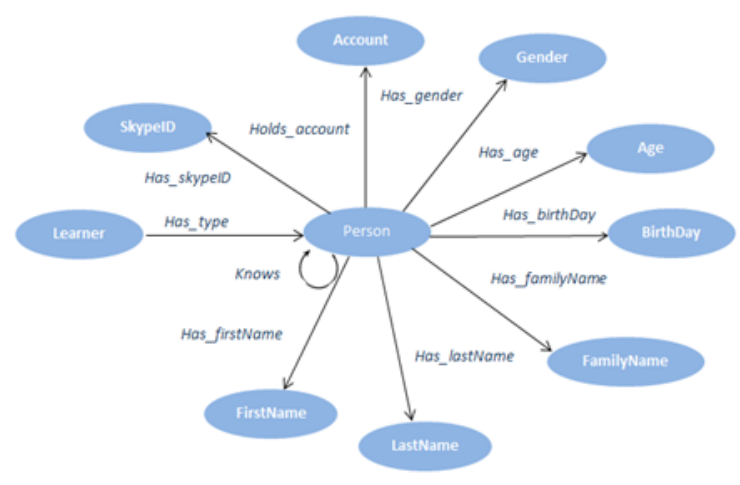

Fig. 3. Learner profile and social interactions Ontology

Figure 4 presents the ontology of the learner's activities. This ontology represents and stores the various information relating to the educational interest and the behavior of the learner. It shows what the learner is interested in, which courses are enrolled in, and what specific activities he has done.

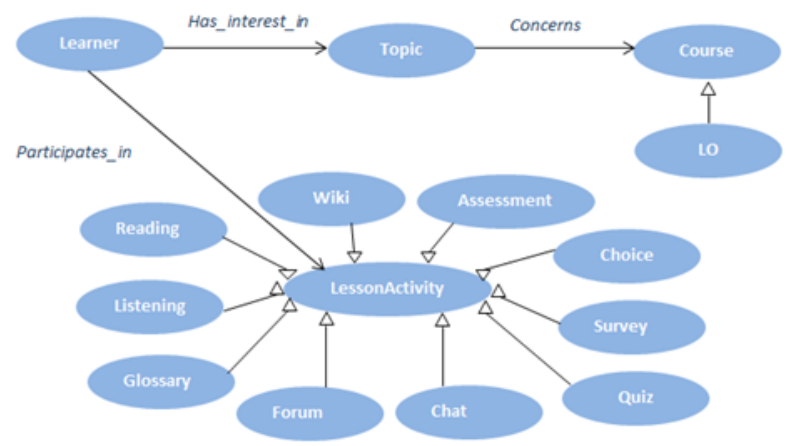

Fig. 4. Ontology of the learner's educational activities

\subsection{Semantic rules}

Semantic Web Rule Language (SWRL) : To consider the learning context in an e-learning system, it is mandatory to represent it. This representation should give a consistent model for gathering and processing the context information. In this work, the LMS ontology is our learning context model. After having built this ontology, it is time now to apply some filtering techniques to deliver to the learner the learning object corresponding to its learning context. The method that we propose to filter learning objects, is to apply a set of business rules, indicating which learning object to recommend in what context. These filtering rules will be used as a recommendation technique because they synthesize the domain knowledge and the constraints that the system must satisfy. Indeed, the business rules are transformed into SWRL (Semantic Web Rule Language) sequences. 
SWRL is a Semantic Web rules language that combines the OWL - DL and OWLLite with the unary/binary sub-language of RuleML (Rule Markup Language). It's useful because it adds expressivity to OWL and Reuses of a set of existent rules. SWRL are structured according to the following schema:

antecedent $->$ consequent.

A rule means $<$ If the antecedent is satisfied then the consequent is also satisfied $>$.

Learning Object Weighting Algorithm: A learning object $l_{i}$ as described in the previous section has nine features according to LOM presentation. Besides these nine features it has the weight feature noted $\mathrm{W}_{\mathrm{i}}$. It represents the relevance of this learning object for the learner in a particular context of learning. $\mathrm{lo}_{\mathrm{i}}=\left\{\mathrm{a}_{\mathrm{i}, 1}, \mathrm{a}_{\mathrm{i}, 2}, \mathrm{a}_{\mathrm{i}, 3}, \mathrm{a}_{\mathrm{i}, 4}, \mathrm{a}_{\mathrm{i}, 5}, \mathrm{a}_{\mathrm{i}, 6}\right.$, $\mathrm{a}_{\mathrm{i}, 7}, \mathrm{ai}_{\mathrm{i}, 8}, \mathrm{a}_{\mathrm{i}, 9}, \mathrm{Wi}$. Since the context of learning is changing, the weight and the relevance of the recommended and delivered learning object are changing. The SWRL rules are used to modify the weight of a learning object. The idea is to reorganize and to filter learning objects following the constraints of the learner in a particular context. Several business rules can be applied to a learning object. Consequently, every new rule modifies and updates the weight of this learning object according to the following equation (Soualah-Alila et al.):

$$
\mathrm{W}_{\mathrm{i}, \mathrm{j}}=\alpha_{\mathrm{j}} \cdot \mathrm{W}_{\mathrm{i}, \mathrm{j}-1}
$$

$\mathrm{W}_{\mathrm{i}, \mathrm{j}}$ is the weight of the learning object loi while applying the business rule $\mathrm{j} . \mathrm{W}_{\mathrm{i}, \mathrm{j}-1}$ is the weight of the learning object lo while applying the business rule $j-1$.

The weight represents the relevance of the learning object in the learning context by respecting the following instructions:

If $\mathrm{W}_{\mathrm{i}}=5$, the learning object has a low relevance for the learning context.

If $\mathrm{W}_{\mathrm{i}}=10$, the learning object has a medium relevance for the learning context.

If $\mathrm{W}_{\mathrm{i}}=20$, the learning object is highly relevant for the learning context.

The value of every Wi is updated while applying every business rule by the object property update_weight. $\alpha_{\mathrm{j}}$ takes values of integers that allow to the weight $\mathrm{W}_{\mathrm{i}, \mathrm{j}}$ to take the values $\overline{5}, 10$ or 20 . Before applying any business rule, the weight $\mathrm{W}_{\mathrm{i}, 0}$ is initialized to $1\left(\mathrm{~W}_{\mathrm{i}, 0}=1\right)$.

The Rules Business Categories: Being delivered the right learning materials is depending on the learning context. This later is a combination of different contextual information that are related to the learner behavior inside the e-learning platform. The logic behind using the Semantic Web Rules Language (SWRL) is to use them as recommendation technique to infer with a reasoning tool the suitable learning materials for the learning context. Since the context of learning is changing according to different contextual information, we distinguish four categories of business rules: Learning History Rules (LHR), Learning Performance Rules (LPR), Learning Social Network Rules (LSNR) and leaning Pathway Rules (PR).

- Learning History Rules (LHR): are used to recommend to the learner the learning materials that are coherent with its learning history. Futures learning materials will be recommended in correspondence with previous materials that are already used by the learner. These rules are equivalent to based-content recommendation techniques used in classical recommendation system. See the following example: 
- Rule: If a learner has used the learning object lo1, he could be interested in the learning object lo2 that belongs to the same subject as lo1. The weight of lo2 is updated to 20 because of its high relevance for the learning context.

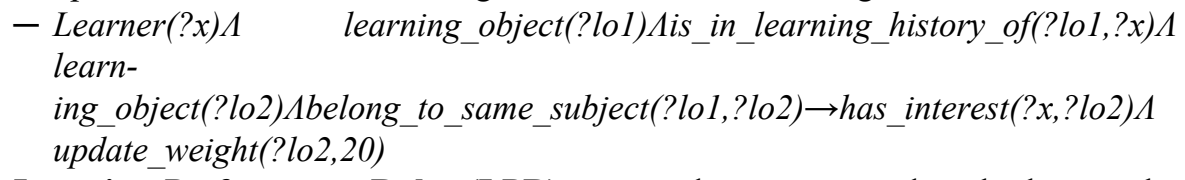

- Learning Performance Rules (LPR): are used to recommend to the learner the learning objects that are coherent with its performance feedback and grades. Futures learning materials will be recommended in correspondence with previous performance while doing lessons activities. See the following example:

- Rule: If a learner has English test score greater than 70, he will access to learning materials of upper intermediate level.

- Learner(?x)Ahas_english_test_level_greaterThan $(? x, 70) \rightarrow$ has_access_to_english_level(? $x$,UpperIntermediate)

- Learning Social Network Rules (LSNR): are used to recommend to the learner the learning objects that are coherent with his social interactions and relationships. The foaf: knows object property mentioned in the previous section is used to visualize the social network of the other learners who have a connection or a relationship with a learner. Futures learning materials will be recommended based on the preferences of his connections. To best filter what are the suitable learning materials to recommend, the connections of the learners should be restricted to learners that have a certain similarity in profiles and learning strategies. These rules are equivalent to collaborative recommendation techniques used in classical recommendation system. See the following example:

- Rule: If a learner $\mathrm{x}$ knows a similar learner $\mathrm{y}$ that has used a learning object lo, he will be recommended the same learning object. The lo weight will be updated to 10 because of its medium relevance.

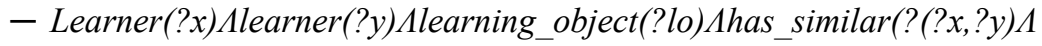
has_learning_object(?y,?lo) $\rightarrow$ has_interest(?x,?lo)4 update_weight(?lo,10)

- Learning Pathway Rules (LPR): are used to determine the order of recommended learning materials in a learning pathway. These rules are important to define the logical sequence of learning object in continued flow. See the following example:

- Rule: The learning object lo1 must be follewed by lo2 and lo2 must be followed by lo3. This an example of a learning pathway.

\section{Conclusion}

There is a rising interest in Context-aware and Context recommendation systems in e-learning. Because they enhance the quality of these systems by helping learners to lead a successful learning experience by recommending them the suitable learning materials. These systems will be more performant and relevant if they take benefits of semantic web technologies. In this paper, we propose a revised version of the 3-tiers e-learning platforms architecture by adding a semantic layer hosting an ontology and 
semantic rules for semantic recommendation systems. The SWRL Rules are used as a recommendation and filtering technique based on learning object relevance. The learning object relevance is determined by the weight value that takes 5 or 10 or 20 . This value changes after every application of SWRL rules. These rules are organised into four categories: Learning History Rules (LHR), Learning Performance Rules (LPR), Learning Social Network Rules (LSNR) and Learning Pathway Rules (PR).

\section{$5 \quad$ References}

[1] B. Bouihi, B and M. Bahaj, "Building An E-Learning System's OWL Ontology By Exploring The UML Model'. Journal of Theoretical and Applied Information Technology, 87(3), 380. 2016

[2] F. Soualah-Alila, F, C. Nicolle, and F. Mendes, "Une approche Web sémantique et combinatoire pour un système de recommandation sensible au contexte appliqué à l'apprentissage mobile", In : 11 ème édition de l'atelier Fouille de Données Complexes, No. 47-58.

[3] M. Grandbastien, B. Huynh-Kim-Bang, and A. Monceaux, "Les ontologies du prototype LUISA, une architecture fondée sur des Web Services Sémantiques pour les ressources de formation". In : 19es Journées Francophones d'Ingénierie des Connaissances, pp. 61-72.

[4] L. Ding, T. Finin, and A. Joshi, "Analyzing social networks on the semantic web". IEEE Intelligent Systems (Trends \& Controversies), 8(6), 815-820.

[5] L. Buriano, L, "Exploiting social context information in context-aware mobile tourism guides". Proceedings of Mobile Guide.

[6] Z. Yu, Y. Nakamura, and S. Jang, S. Kajita, K. Mase, "Ontology-based semantic recommendation for context-aware e-learning". Ubiquitous Intelligence and Computing, 2007, 898-907. https://doi.org/10.1007/978-3-540-73549-6 88

[7] A. Schmidt, and C.Winterhalter, C, "User context aware delivery of e-learning material: Approach and Architecture'. Journal of Universal Computer Science, 2004, 10(1), 28-36.

[8] M. PVillalon, M.C Suárez-Figueroa, R. García-Castro, and A. Gómez-Pérez, "A context ontology for mobile environments', 2010.

[9] R. Benlamri, R and X. Zhang, "Context-aware recommender for mobile learners". Humancentric Computing and Information Sciences, Springer, 2014, 4(1), 1-34. https://doi.org/10. 1186/s13673-014-0012-Z

[10] Z. Yu, Z , Y. Nakamura, S. Jang, a S. Kajita, and K. Mase K, "Ontology-based semantic recommendation for context-aware e-learning". Ubiquitous Intelligence and Computing, 2007, 898-907. https://doi.org/10.1007/978-3-540-73549-6 88

[11] L.P Shen, and R.M Shen, "Ontology-based learning content recommendation". International Journal of Continuing Engineering Education and Life Long Learning, 2005, 15(3-6), 308-317. https://doi.org/10.1504/ijceell.2005.007719

[12] N. Henze, and P. Dolog, and W. Nejdl, "Reasoning and ontologies for personalized elearning in the Semantic Web", Educational Technology \& Society, 2004, 7(4), 82-97.

[13] S. Shishehchi, S.Y, Banihashem, and N.A.M, Zin, "A proposed semantic recommendation system for e- learning: A rule and ontology based e-learning recommendation system". In Information Technology (ITSim), IEEE 2010 International Symposium, 2010 , Vol. 1, pp. 1-5. https://doi.org/10.1109/itsim.2010.5561329

[14] F. Soualah-Alila, N. Christophe N, and M. Florence, "Context-Aware Adaptive System For M-Learning Personalization". IE14 13 th International Conference on Informatics in Economy Education, 2014. 


\section{Authors}

Bouchra Bouihi is with University Hassan $1^{\text {st }}$, Faculty of Sciences \& Technology, Settat, Morocco (bouchrabouihi@gmail.com).

Mohamed Bahaj is with University Hassan $1^{\text {st }}$, Faculty of Sciences \& Technology, Settat, Morocco (mohamedbahaj@gmail.com).

Article submitted 2019-03-27. Resubmitted 2019-05-05. Final acceptance 2019-05-28. Final version published as submitted by the authors. 